\title{
LA CONCEPCIÓN DEL ESPACIO-TIEMPO EN LA COSMOLOGÍA SARVĀSTIVĀDA
}

\author{
Juan Arnau \\ Instituto de Historia de la Ciencia y de la Medicina López Piñero (CSIC-Universidad de Valencia) \\ arnaujuan@gmail.com
}

Recibido: 28 junio 2011; Aceptado: 16 marzo 2012.

Cómo citar este artículo/ Citation: Arnau, Juan (2013), "La concepción del espacio-tiempo en la cosmología sarvāstivāda”, Asclepio 65 (1): p006. doi: http://dx.doi.org/10.3989/asclepio.2013.06

RESUMEN: El presente artículo se orienta a la reconstrucción de los modelos cosmológicos de la escuela de Cachemira (s. IV), registrados en el compendio de la escuela sarvāstivāda-vaibhāsika titulado Abhidharmakośabhāsya, atribuido al monje budista Vasubandhu. Se identifican algunas de las fuentes textuales que dieron lugar a estos modelos y se describe con detalle su estructura general, compuesta de tres ámbitos (sensible, materia sutil e inmaterial). Se analizan algunas de las características más relevantes del espacio y del tiempo y su relación con la vida consciente, descubriéndonos unos modelos cosmológicos inéditos entre sus contemporáneas grecolatinos.

PALABRAS CLAVE: Cosmología; Abhidharmakośabhāsya; Sarvāstivāda; Espacio; Tiempo.

\section{SPACE AND TIME CONCEPTIONS IN SARVĀSTIVĀDA COSMOLOGY}

ABSTRACT: The present article focuses on the reconstruction of the cosmological models from the school of Kashmir (fourth century), as they are registered in the compendium of sarvāstivāda-vaibhāsika school, attributed to the Buddhist monk Vasubandhu and titled Abhidharmakośabhāsya. The articles identifies the different textual sources of the cosmological models and describes its general structure, based in three realms (sensible, subtle matter and immaterial). It analyzes the relevant characteristics of the concepts of Space and Time, discovering and unusual model in comparison with the contemporaries cosmologies from the Mediterranean.

KEY WORDS: Cosmology; Abhidharmakośabhāsya; Sarvāstivāda; Space; Time.

Copyright: () 2013 CSIC. Este es un artículo de acceso abierto distribuido bajo los términos de la licencia Creative Commons Attribution-Non Commercial (by-nc) Spain 3.0. 


\section{INTRODUCCIÓN}

En torno al siglo IV, en los tratados del abhidharma de Cachemira, encontramos uno de los primeros registros por establecer una cosmología coherente en India. Este mapa del tiempo y del espacio se encuentra esbozado en un tratado enciclopédico titulado Abhidharmakośa (Kośa, en adelante), atribuido al monje budista Vasubandhu. En él se recogen las teorías fundamentales sobre el cosmos de la escuela sarvāstivāda-vaibhāsika. El tratado, escrito en sánscrito, incorpora un comentario (bhāsya) del propio Vasubandhu (Vallée-Poussin, 1988).

La cosmografía del Kośa concibe un número incalculable (sāhaśra) de universos. Todos ellos comparten una estructura similar, denominada técnicamente cakravāda. Dicha estructura se organiza verticalmente en tres niveles o ámbitos cósmicos (dhātu), cada uno de los cuales comprende diversos planos de existencia (bhūmi). Todos los universos carecen de límite espacial y temporal. La característica principal de este modelo cosmológico consiste en que los diferentes ámbitos en los que se estratifica cada universo particular corresponden a los diversos estados mentales, que llevan aparejados diferentes morfologías y modos de ser. De modo que cada uno de estos "planos" no se entienden como localizaciones en sentido estricto, sino más bien como temperamentos o estados de ánimo, siendo el propio universo indisociable de la vida mental de aquellos que lo habitan.

La distribución en el espacio y la evolución en el tiempo del cosmos no se articula mediante modelos geométricos, como en el Almagesto, ni mediante fuerzas impersonales y concéntricas, como la gravedad en la cosmología contemporánea, sino en virtud de las excentricidades de la vida consciente. De modo que los destinos individuales de los seres no se encuentran a merced del destino del universo, que prepara las condiciones para su aparición o garantiza su supervivencia, sino que son sus propias acciones, con sus estados mentales asociados, las que trazan el mapa y el calendario cósmicos.

El abanico de las experiencias conscientes, de lo tosco a lo sutil, de lo instintivo a lo cultivado, configura tanto el espacio de la cosmografía como el tiempo y periodicidad de los ciclos de despliegue y repliegue del universo. Algunos investigadores han visto en estas asociaciones entre mente y universo "la contraparte poética, imaginativa y mítica de las descripciones de los estados meditativos (jhāna)" (Gethin, 1997, p. 186). En lo que sigue veremos que la propia escolástica no se posiciona claramente sobre si ese paralelismo debe de entenderse literal o metafóricamente. La mente es a veces metáfora del cosmos, y el cosmos metáfora de la mente, pero en ciertas ocasiones se establece entre ambos un paralelismo o complementariedad que parece afirmar la existencia independiente de ambas entidades. Sea como fuere, comparar las descripciones de los estados de la mente con narraciones de naturaleza cosmogónica permitirá comprender con mayor amplitud la idea del cosmos de los antiguos budistas (Gethin, 1997, p. 189).

En la jerarquía espacial, que es al mismo tiempo una jerarquía de sensibilidades hay, en sentido ascendente, un ámbito del deseo (kāmadhātu), un ámbito de materia sutil (rūpadhātu) y un ámbito inmaterial (arūpyadhātu). Cada uno de ellos se subdivide en diversos planos de existencia (bhūmi), hasta completar treinta y uno.

\begin{tabular}{|c|c|}
\hline $\begin{array}{c}\text { Ámbitos cósmicos } \\
\text { (dhātu) }\end{array}$ & $\begin{array}{c}31 \text { niveles de existencia } \\
\text { (bhūmi) }\end{array}$ \\
\hline $\begin{array}{l}\text { Inmaterial } \\
\text { Ārūpadhātu }\end{array}$ & 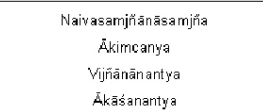 \\
\hline \multirow{5}{*}{$\begin{array}{l}\text { Materia sutil } \\
\text { Rüpadhātu }\end{array}$} & $\begin{array}{l}\text { Akaristha } \\
\text { Sudarsana } \\
\text { Sudrša } \\
\text { Aviha }\end{array}$ \\
\hline & $\begin{array}{c}\text { Asankifasatta } \\
\text { Brhatphala }\end{array}$ \\
\hline & $\begin{array}{l}\text { Sublha-kinha } \\
\text { Appamannubha } \\
\text { Parittasubha }\end{array}$ \\
\hline & $\begin{array}{l}\text { Ābhāsvara } \\
\text { Apramănāabha } \\
\text { Parittābha }\end{array}$ \\
\hline & $\begin{array}{l}\text { Mahābruahmā } \\
\text { Brahmā purohita } \\
\text { Brahmākāyika }\end{array}$ \\
\hline \multirow{3}{*}{$\begin{array}{l}\text { Sensual } \\
\text { Kămadhātu }\end{array}$} & 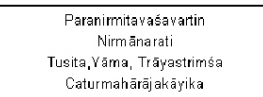 \\
\hline & Manusya \\
\hline & $\begin{array}{c}\text { Asura Preta } \\
\text { Tiryagyoni Naraka }\end{array}$ \\
\hline
\end{tabular}




\section{LA CUESTIÓN COSMOLÓGICA EN LOS NIKĀYA}

Esa actitud reacia a desligar la cosmología de las facultades y procesos mentales es herencia de la literatura de los nikāya. La diferencia de niveles, el salto entre lo literal y lo metafórico, pierde en estas narraciones la escala de valores que acostumbra a tener en las disciplinas científicas modernas. El primer diálogo del Dìghanikāya, con un claro talante empírico-racional, critica las diversas creencias, falsas y desaconsejables, relacionadas con la predicción de futuro ${ }^{1}$. El arte de los hechizos, presagios y augurios mediante el análisis de los sueños, las líneas de las manos, las roeduras de ratones o diversos tipos de oblaciones en el fuego. Todas estas prácticas se consideran bajas artes, medios de vida equivocados, formas ruines de ganarse el sustento que se sirven del engaño a ingenuos y sentimentales. Se alude también los oráculos y a aquellos que ven signos por doquier y predicen con ellos lo que vendrá. Entre estas bajas artes se menciona además a aquellos que predicen eclipses y tempestades, caídas de meteoros y temblores de tierra, incendios cósmi$\cos$ y desviaciones del curso natural de los astros. $Y$ se desaconseja al monje que no se ocupe de estos asuntos, siendo propios de mentes dominadas por el deseo, recordando que el propio Buda fue reacio a debatir cuestiones cosmológicas, por considerarlas especulaciones que desvían la atención de lo verdaderamente fundamental. Dichas actividades no sólo no contribuyen, sino que obstaculizan la erradicación de la ignorancia y el deseo ciego: eje en torno al cual debe el monje organizar su vida mental. ${ }^{2}$

Desde esta perspectiva puede hablarse de una actitud anticosmológica en el budismo temprano. Sin embargo, esta tendencia antigua no se mantendrá en la escolástica posterior y, con el tiempo, se desarrollarán sistemas que expliquen el universo en función de su estructura espacial y temporal. Siguiendo una inclinación muy característica india: la asimilación frente al descarte; muchas de las concepciones budistas sobre el universo se encuentran ya en el periodo védico y serán incorporadas y adaptadas posteriormente por el abhidharma. La influencia de su cosmología se extenderá, desde el norte de India hasta las tradiciones mahāyāna de Tíbet y Asia oriental. Mientras que el abhidharma theravāda predominará en el Sur de India (Sri Lanka) y el Sudeste asiático. Aunque existen diferencias menores, las cosmologías abhidhármicas comparten, de manera general, cinco premisas:

1. El universo ha existido siempre y siempre existirá. Carece de límite temporal, pudiéndose obviar la caracterización de su creador y la elaboración de una cosmogonía.

2. El universo carece de límite espacial.

3. La ley de la causalidad (que no se limita al ámbito material) rige el funcionamiento del mundo. Esa ley se expresa de manera general mediante el dictum: asmin sati idam bhavati (dado esto ocurre aquello) y de manera particular, referida a los seres conscientes, mediante la doctrina del origen condicionado (pratityasamutpāda).

4. La fuerza gravitante de las acciones de los seres (toda acción lleva asociado un estado mental) configura la estructura del universo, tanto en el espacio como en el tiempo.

5. El universo se estructura en diferentes ámbitos de existencia (según el principio anterior) que constituyen una jerarquía. Dichos ámbitos se encuentran asociados a un modo especial de proceder adquirido por la repetición de actos, tendencias instintivas e inclinaciones del pensamiento. Todos ellos constituyen los estados mentales dominantes (aunque no exclusivos) de cada ámbito.

\section{ESPACIO, IDENTIDAD Y DIVERSIDAD}

Para entender correctamente estos modelos cosmológicos es necesario referirnos previamente a las concepciones budistas de la vida consciente. Según éstas, en la sucesión en el tiempo y el espacio, en su duración y movimiento, los seres ofrecen diferentes versiones de sí mismos, se trasmutan y se rehacen y únicamente lo actuado puede cambiar la identidad de las cosas. Ese es el drama del que surge la cosmología budista. Un drama en el que el sujeto se define como una particular unidad de actos dentro de un proceso continuo de percepción cognitiva (vijñānasamtāna), que recorre diversas formas de existencia. Los seres se transforman unos en otros, metamorfoseándose según ganen o pierdan en inteligencia o estupidez. El universo no se mueve por fuerzas impersonales, sino por las excentricidades de la vida consciente. Los destinos individuales no se encuentren a merced de la evolución ciega de la materia, sino que son las propias acciones de los seres, las que trazan la singladura de la nave del mundo. El tejido del espacio-tiempo es suplantado por una matriz en cuyo molde los seres nacen de sus propias acciones. $Y$ dado que todo acto lleva un estado mental asociado, el conjunto de los estados mentales que forman el temperamento o personalidad, se encontrará asociado con los diferentes ámbitos cósmicos.

La variedad y riqueza de la experiencia humana, capaz de experimentar los dolores más abyectos y las afinidades más sublimes, sitúa al hombre en una posición privilegiada en todo este entramado. Dentro de esas capacidades para la acción, la tradición budista establece una jerarquía de los seres en función de su sensibilidad. En este sentido, el mapa del cosmos es, para el budismo, un detallado informe de todas las 
experiencias posibles (de todos los estados mentales) por las que habrán de pasar los seres en su deambular (errático o intencional) por la existencia (samsāra). Hay aquí un cambio radical de perspectiva: El espacio y sus ámbitos de vida son una creación del temperamento de los seres que los habitan. El espacio ya no es una categoría preconceptual que sirve de escenario para el drama de la vida consciente, sino que es una consecuencia de esa misma vida, que se crea un hueco donde habitar.

Una onda de gravedad mantiene el pulso del mundo. La diversidad del universo material y espiritual tiene en efecto una causa y en modo alguno puede considerarse resultado del azar. Al inicio de cada ciclo el universo se recrea, y el sujeto de ese "se" impersonal, lo que pone en marcha de nuevo el mundo, es la fuerza del karma, la integral de todas las acciones de los seres conscientes. La célebre fórmula: "la diversidad del mundo procede de la acción" (karmajam lokavaichitryam), citada por Vasubandhu en el Kośa sirve de justificación al sentido y configuración del cosmos (Vallée-Poussin, 1988, p. 551). ${ }^{3}$ Dicha fórmula contiene, como afirma Louis de la Vallée Poussin, el alfa y el omega de lo que el monje debe saber acerca del mundo (Vallée-Poussin, 1908, p. 130). Se trata de uno de uno de los pilares en los que se apoya la tradición budista y cuya dimensión no es exclusivamente moral, sino también cosmológica: el mundo material se encuentra organizado por la retribución de los actos conscientes y por ambientes en los que predominan determinadas actitudes y hábitos mentales. Este factor hace posible que, en determinadas circunstancias, mediante el ejercicio de la meditación, sea posible experimentar ámbitos más allá del propio en el que se vive. De manera general, los seres se dividen en aquellos que habitan el ámbito sensual (kCEmavacara), aquellos que habitan el ámbito de la materia sutil (rūpavacara) y aquellos que habitan el ámbito inmaterial (arūpavacara)

Es muy posible que tanto el budismo popular como el escolático hicieran posibles ambas lecturas: espacios cósmicos y estados de la mente. Un ejemplo claro de esta combinación entre crítica y asimilación lo tenemos en el Kevaasutta del DïghanikCEya. ${ }^{4}$ En este relato el deseo de comprender el espacio como ubicación (bhājanaloka) se representa en oposición al conocimiento y profundización del espacio como estado de conciencia (sattvaloka).

\section{EL MUNDO SENSIBLE}

El libro tercero del Kośa sintetiza las nociones escolásticas en torno a la naturaleza del espacio y del tiempo, su estructura y funcionamiento. El espacio cósmico se encuentra estratificado en tres niveles, que son al mismo tiempo tres estados de la mente consciente.
El términos espaciales los niveles son únicamente dos pues, como veremos, el tercero (arūpyadhātu) es inmaterial y no puede considerarse espacial, aunque sí temporal. Hay un tiempo sin espacio, que permanece entre los ciclos de contracción y despliegue del cosmos.

En el nivel inferior se encuentra el mundo sensual, de tosca materialidad, denominado kāmadhātu (Vallée-Poussin, 1988, p. 365)..$^{5}$ Es el ámbito de la experiencia sensible e incluye cinco ámbitos del renacer (gati). ${ }^{6}$ El mundo del deseo sensual es el mundo que todos conocemos. Se trata de un mundo gobernado por el deseo (kāma). Y ¿qué es kāma?, se pregunta Vasubandhu. Kāma es apetito de placer, impetuoso deseo de alimento, deseo sexual. Kāma es "aquello que desea" (kāmyata aneneti kāmah). Y se cita una sentencia de Śāriputra, que nos recuerda que el budismo tuvo su origen en las tradiciones ascéticas: "Las cosas buenas de este mundo no son kāma; en los humanos, kāma es el deseo que nutre la imaginación. Poca importancia tienen los objetos del deseo: el sabio es indiferente a ellos". ${ }^{7}$ El comentario define también el compuesto kāmadhātu. Dhātu es aquello que porta (dadhāti) una característica propia (svalaksana), en este caso kāma (la sensualidad) y sus objetos de deseo (kāmaguna). De este modo, kāmadhātu es el dhātu asociado con kāma y lo traducimos por "mundo sensual". Del mismo modo que rūpadhātu significa el dhātu asociado con la materia sutil (rūpa). Rūpa es también aquello que es susceptible de ofrecer resistencia (rūpana) debido a su consistencia. Mientras que ārūpa ("inmaterial”) define el tercer ámbito, el dhātu carente de materia. ${ }^{8}$

\section{EL MUNDO SUTIL}

El término rūpa generalmente hace referencia a la materia que conforma y se manifiesta a los sentidos. ${ }^{9}$ Pero en el contexto de las teorías sobre la mente y el cosmos, asociado con dhātu en el compuesto rūpadhātu, se refiere al segundo de los tres niveles que configuran tanto el cosmos como la experiencia mental. Rūpadhāthu, que puede traducirse como "mundo de la materia sutil", se compone de diecisiete ámbitos (bhūmi), cuyo material se asocia con los diferentes estadios de la meditación (dhyāna) (Vallée-Poussin, 1988, pp.365-366), ${ }^{10}$ de ahí que dichos ámbitos puedan experimentarse tanto renaciendo en ellos como mediante el cultivo mental. Se habla de "materia sutil" por su naturaleza serena y contemplativa, tanto en los ámbitos cósmicos como en sus estados mentales asociados con ellos. En rūpadhātu la actividad sexual, táctil, olfativa y gustativa han desaparecido, así como cualquier tipo de dolor físico (aunque no ciertas inquietudes o insatisfacciones debidas a la fugacidad de la experiencia), quedando sólo operativos lo visual y auditivo. 
¿Cual es la naturaleza y alcance de estos estados de la mente? El término dhyāna se refiere al trance o absorción meditativa. La tradición distingue cuatro de estos trances, los primeros tres pertenecen al ámbito de rūpadhātu, mientras que el cuarto puede pertenecer tanto al mundo de materia sutil como al mundo inmaterial de puro entendimiento (arūpyadhātu). ${ }^{11}$ Los dhyāna tienen como prerrequisito la concentración mental (śamatha). En ellos la actividad sensorial queda en suspenso y la actividad de la mente se atenúa progresivamente. Así, en el primer dhyāna se dan todavía la conceptualización (vitarka) y la reflexión (vicāra), mientras que en el segundo han desaparecido. En el cuarto dhyana es donde se adquiere el conocimiento de las vidas previas y ciertos poderes extraordinarios, quedando extinguida toda sensación y obteniéndose una ecuanimidad que da acceso a los estados del mundo inmaterial..$^{12}$ La experiencia de los dhyāna no constituye un fin en sí mismo, sino sólo un medio para la obtención del nirvana. Se consideran estados pasajeros y condicionados, y por tanto insatisfactorios. Los trances no suponen una transformación mental definitiva, una vez se sale de ellos la mente recobra las carencias que tenía antes de entrar en ellos. La asociación entre estados de la mente (citta) y ámbitos cósmicos (bhūmi) permite experimentar estos ámbitos en largos periodos de tiempo, cuando se renace en ellos, o mediante los vislumbres pasajeros de la meditación, que pueden entenderse como estados de paramnesia pues, como veremos, todos los seres ya han recorrido los diversos ámbitos en numerosas ocasiones (Vallée-Poussin, 1988, pp.365). ${ }^{13}$ Dicha vinculación explica que un mismo término, bhūmi, se utilice tanto para referirse a los diferentes niveles de evolución espiritual del individuo como a los diferentes lugares del espacio cósmico. La jerarquía cosmográfica corre en paralelo a la jerarquía de la experiencia mental. El viaje de la conciencia puede entenderse como un viaje cósmico, cuyo desplazamiento es posible gracias al cultivo de la mente.

\begin{tabular}{|c|c|c|}
\hline \multicolumn{2}{|c|}{ Ámbitos cósmicos (dhātu) } & Estados mentales (citta) \\
\hline 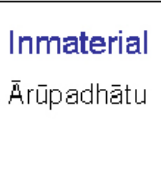 & $\begin{array}{c}\text { Naivasamjñnānāsamjña } \\
\text { Ākimcanya } \\
\text { Vijñānānantya } \\
\text { Ākāśanantya }\end{array}$ & \multirow[t]{3}{*}{ Cuarto dhyāna } \\
\hline \multirow{5}{*}{$\begin{array}{l}\text { Materia } \\
\text { sutil } \\
\text { Rūpadhātu } \\
\text { (brahmas) }\end{array}$} & $\begin{array}{l}\text { Akanistha } \\
\text { Sudarśana } \\
\text { Sudrśa } \\
\text { Awrha }\end{array}$ & \\
\hline & $\begin{array}{l}\text { Asañnasatta } \\
\text { Brhatphala }\end{array}$ & \\
\hline & $\begin{array}{l}\text { Subha-kinha } \\
\text { Appamānsubha } \\
\text { Parittasubha }\end{array}$ & Tercer dhyāna \\
\hline & $\begin{array}{l}\text { Ābhāsvarà } \\
\text { Apramānābha } \\
\text { Pạrttāăbhă }\end{array}$ & Segundo dhyāna \\
\hline & $\begin{array}{c}\text { Mahābrahmā } \\
\text { Brahmāpurohita } \\
\text { Brahmākāyika }\end{array}$ & Primer dhyāna \\
\hline \multirow[t]{3}{*}{$\begin{array}{l}\text { Sensual } \\
\text { Kāmadhātu }\end{array}$} & $\begin{array}{c}\text { Paranirmitawaśavartin } \\
\text { Nirmānarati } \\
\text { Tusita,Yāma, Trāyastrimśa } \\
\text { Caturmah ārājakāāyika }\end{array}$ & \multirow[t]{2}{*}{$\begin{array}{c}\text { Estados dominados por el desapego, la } \\
\text { generosidad y la virtud }\end{array}$} \\
\hline & Manusya & \\
\hline & $\begin{array}{l}\text { Asura Preta } \\
\text { Tiryagyani Naraka }\end{array}$ & $\begin{array}{l}\text { Estados dominados por el odio, la } \\
\text { confusión y el deseo ciego }\end{array}$ \\
\hline
\end{tabular}




\section{EL MUNDO INMATERIAL}

El terceroymás elevado de los mundos, ārūpyadhātu, no es un lugar en el espacio pero sí un momento en el tiempo. ${ }^{14}$ No es lícito hablar aquí en términos espaciales pues los dharma inmateriales no ocupan lugar. $Y$ sin embargo este estado de la mente tiene cuatro formas de existencia. ${ }^{15}$ En este contexto "existencia" significa la aparición de los skandha (proyectados por el karma) en un nuevo ámbito. Vasubandhu pregunta: "Si los estados mentales tienen como soporte la materia (rūpa), ¿cuál será el soporte de la serie de estados mentales (que constituyen los seres) en ārūpyadhātu?". La respuesta, en la siguiente estrofa, no carece de hermetismo: adjudica el soporte al género y a las facultades vitales. ${ }^{16}$ En comentario añade que los seres de arūpyadhātu encuentran soporte en dos dharma disociados de la mente: nikāyasabhāgatā (género o clase) y jīvitendriya (facultad vital) (ValléePoussin, 1988, p. 219). ${ }^{17}$ Sabhāga hace referencia a la noción de participación. Sabhāgata es la causa del resemblar, aquello que produce el parecido entre los seres vivos. Pero, en el ámbito de las ideas (buddhi) y de las expresiones (prajñapti), tiene relación también con lo genérico, con aquello que se aplica a diferentes entidades. Así sabhāgata da sentido o justifica expresiones como skandha o dhātu, que tienen una naturaleza genérica. Las resonancias platónicas son más que evidentes. En el mundo material esto no sería posible porque estos dos dharma carecen de la fuerza necesaria para mantener, por sí mismos, la existencia. Pero en el mundo inmaterial sí que es posible que la idea o género se mantenga viva, y que la vida a su vez mantenga la idea. El género o idea y la facultad vital se mantienen uno a otro en ārūpyadhātu.

En otro lugar de la misma obra se vuelve a plantear la cuestión. ${ }^{18}$ ¿Cómo puede existir la mente en ārūpyadhātu sin que haya un soporte material?, o, ¿cómo podría algo inmaterial dar con imágenes, intereses o categorías (nikāya)? Si la mente no se ocupara de nada, no sería consciente (la consciencia es siempre de algo), y si se ocupara de todo lo posible, no sería la mente de un ser particular con su propio itinerario kármico. Se concluye de nuevo que su soporte ha de ser el género (nikāya) y la vida (jivita).

La escuela sautrāntika no admite la existencia de este dharma genérico. La serie de estados mentales carece de un soporte externo a ella misma en arūpyadhātu. ¿Cómo es posible que exista sin soporte? Simplemente porque es "proyectada" mediante una determinada causa (karmakleśa). Si dicha causa no se encuentra libre del apego por la materia, la serie reaparecerá en el mundo material y encontrará soporte en la materia. En caso contrario, la mente (considerada una serie de estados mentales) existirá sin relación a la materia. Al margen del modo en el que se justifique un mundo mental sin soporte material, lo significativo desde la perspectiva cosmológica es la hipótesis misma de un ámbito inmaterial. A ese ámbito, como veremos, quedará reducido el universo en sus periódicos ciclos de repliegue.

Vasubandhu sugiere que estos tres dhātu forman tres niveles, tanto en la jerarquía cósmica como en la evolución de las consciencias, pero que estas "capas" se encuentran de alguna manera entretejidas. El filósofo cuestiona si debería considerarse "integral" a un dhātu, es decir, del dominio de ese dhātu, a todos los dharma que se producen en él. Y concluye aceptando la posibilidad de que algunos dharma producidos en kāmadāthu sean de la esfera de rūpadhātu o arūpyadhātu, por ejemplo debido a los logros de la concentración mental. En el libro octavo se añade que estos estados (dhyāna) tienen siempre un recorrido ascendente (y no descendente), pues de otro modo carecerían de utilidad. Es decir, los dhyāna de otras esferas se producen siempre en estados de conciencia que pertenecen a esferas inferiores (Vallée-Poussin, 1988 , p. 1250). ${ }^{19}$ Por lo tanto, no todos los dharma producidos en un dhātu tiene el anhelo ( $r \bar{g} g a)$ propio de ese dhātu. El anhelo propio de kāmadhātu es el anhelo de seres absorbidos (samāhita) por su ambiente y no desapegados de los deseos que su mundo ofrece. Lo mismo puede decirse para los otros dos ámbitos (rūpadhātu y arūpyadhātu), pero ello no significa que no puedan producir ciertos dharma pertenecientes a estadios superiores (del cosmos o de la mente).

Algunos textos conciben la estructura de los dhātu en capas horizontales planas, no habiendo discontinuidad en ninguna dirección de los cuatro puntos cardinales. ${ }^{20}$ Otras opiniones (Sthiramati) describen estos ámbitos como esféricos, extendiéndose no sólo en la dirección de los puntos cardinales sino también hacia el zenit y el nadir. Aunque cada una de estas capas pertenece a un universo concreto, quienquiera que se desapega de un kāmadhātu particular se desapega al mismo tiempo de los kāmadhātu de todos los universos, y lo mismo puede decirse para los otros dhātu. Sin embargo, los poderes extraordinarios obtenidos mediante el primer dhyāna se limitan al universo en el que se producen.

En los ámbitos inmateriales la calma es grande y la intelección pequeña, lo contrario que en otros ámbitos (Vallée-Poussin, 1988, pp. 1215-1216). ${ }^{21}$ El primer dhyāna se encuentra asociado con la comprensión y discriminación (vicāra), la satisfacción afectiva (prīti) y la dicha (sukha), elementos que irán desapareciendo en los subsiguientes dhyāna. En el segundo dhyāna se elimina la discriminación, quedando unidamente la satisfacción afectiva y la dicha. En el tercero sólo permanece la dicha. En el cuarto desaparece esta última. ${ }^{22}$ 
"Los ārūpya son como los dhyāna en número y naturaleza. Hay cuatro ārūpya y cada uno es doble: son estados de la mente concentrada y ámbitos de existencia. Están constituidos por cuatro skandha, pues la materia (rūpa) se encuentra ausente en ellos". ${ }^{23} \mathrm{El}$ tratado intenta explicar en qué sentido ārūpyadhātu, que no es en realidad ningún lugar ni estadio, es cuádruple desde la perspectiva de los seres que allí renacen, que son "aquellos que han dominado la idea de la materia". Los seres que moran en ārūpyadhātu carecen de visión y oído (a diferencia de los de rūpadhātu) y por tanto de la percepción cognitiva asociada a estos órganos. ${ }^{24}$

A continuación se discuten las diferentes posturas de las escuelas acerca de la posibilidad de un ámbito inmaterial. Algunas sostienen que ārūpyadhātu no es completamente inmaterial sino que se compone de una materia extremadamente sutil, transparente, similar a la de los diminutos y prácticamente invisibles seres acuáticos de los que habla el vinaya, que apenas puede percibirse. Los argumentos para mantener esta tesis son cuatro: (1) El calor y la vida se encuentran asociados. (2) Se dice que lo material se apoya en lo inmaterial, y a la inversa (nāma, los cuatro skandha no materiales, y la materia se apoyan mutuamente). (3) Además, los skandha materiales e inmateriales son un efecto de la percepción cognitiva (vijñāna). Y finalmente se sostiene que (4) la percepción cognitiva no es independiente de la materia física, la sensación, las ideas o los hábitos. Sin embargo, estos argumentos no resultan convincentes. Prueba de ello es que cuando los sūtra hablan de la vida (āyus) asociada con el calor (que es material), o cuando hablan de que lo material se apoya en lo inmaterial y viceversa, no se refieren a todos los tipos de vida sino sólo a aquellos que surgen en el ámbito del deseo y el ámbito de la materia sutil. Respecto al tercer argumento, hay que distinguir entre las percepciones cognitivas producidas por las samskāra de kāmadhātu y rūpadhātu, de aquellas producidas por las saxskāra de ārūpadhātu. Respecto al cuarto, el sūtra niega que la percepción cognitiva se mueva independientemente de los vijñānasthiti, es decir, al margen de la materialidad, la sensación, las ideas y la intención (rūpa, vedāna, samjñā y samskāra), pero habría que dilucidar a qué ámbito pertenecen estos, pues bien podrían ser consecuencia de retribuciones kármicas de un ámbito material.

Se concluye que en el mundo de ārūpya toda sensación (vedanā) ha sido dejada atrás y que se trata de un mundo inmaterial. ${ }^{25}$ Cuando un ser logra renacer en este ámbito, su propia materialidad (rūpa) queda en suspenso durante largos periodos de tiempo. Se pregunta entonces de dónde extrae dicho ser su cuerpo cuando renace en un ámbito inferior. Se responde que la materia física surge de la mente $(\mathrm{citta}) .{ }^{26}$ Una acción del pasado puede "materializarse" mediante la traza ( $v \bar{a} s a n \bar{a})$ que dejó en la mente y dicha maduración explica la emergencia de lo material a partir de lo mental. Samghabhadra (Prakaranaśāsana) comenta: "Cuando un ser muere en ārūpyadhātu y renace en un ámbito inferior, la serie continua de sus estados mentales (cittasamtati) produce la materia (se fabrica un cuerpo), así es como de la mente puede surgir la materia. Nosotros mantenemos que en este mundo, los dharma materiales e inmateriales se producen de hecho en mutua dependencia. Las transformaciones de lo mental producen la diversidad de lo material. Cuando se transforman o evolucionan los órganos de los sentidos, que son materiales, las percepciones cognitivas difieren. Además. Éste no es el único modo de surgimiento de lo material, también puede originarse a partir de procesos en ámbitos materiales."

Los ārūpya surgen como consecuencia del desasimiento de un estado anterior, Cada uno de ellos recibe su nombre de los ejercicios preparatorios que facilitan el acceso a los mismos: ākāśantya, vijñānānantya y ākimcanya. El viajero y filósofo chino Xuanzang añade que, en sus ejercicios, el meditante debe pensar sucesivamente: "El espacio (ākāśa) es infinito", "la percepción cognitiva (vijñāna), en sus seis modos, es infinita", "poco es lo que hay (ākimcanya)". ${ }^{27}$ El locus classicus al que hace referencia es un pasaje del Culasuññata-sutta, donde el Buda habla de "permanecer en la plenitud de la vacuidad". ${ }^{28}$ El texto describe las tres fases mencionadas: la atención al espacio ilimitado, la atención a la infinitud de la percepción cognitiva, que nunca se interrumpe (simplemente cambia de modo y escenario en función del itinerario kármico que la guía), y la atención a la unidad de la esfera de la nada. En cada una de estas fases la mente consigue clarificarse, equilibrarse y liberarse gradualmente hasta finalmente alcanzar el último de los ārūpya: nasamjñānāpyasamjñāka.

\section{EL REPLIEGUE CÓSMICO}

En el capítulo dedicado a los diferentes tipos de conocimiento directo, dentro de la sección dedicada al recuerdo consciente de vidas anteriores, ${ }^{29}$ Buddhagosa describe los procesos de expansión y contracción que experimenta el "sistema de mundos" (lokadhātu), en cada uno de los cuales se distinguen 31 ámbitos de existencia (bhūmi). La contracción se produce al mismo tiempo en cúmulos que comprenden innumerables mundos. ${ }^{30} \mathrm{En}$ el caso de los periodos de contracción, se trata de eras de disminución, mientras que en los periodos de expansión de trata de eras de incremento. ${ }^{31}$ Aquello que reemplaza la contracción se encuentra enraizado en ella y lo mismo puede decirse de lo que incrementa la expansión. ${ }^{32}$ Existen tres clases de periodos de contracción, los desencadenados por el fuego, por el agua, y por el viento. Hay, al mismo tiempo, tres límites superiores a dicha contrac- 
ción: la contracción por el fuego puede arrasar todos los ámbitos de existencia que quedan por debajo de ābhassara, la contracción por el agua tiene su límite en el ámbito de subhakinha, y la contracción por el viento tiene su límite en el ámbito vehapphala. ${ }^{33}$

Como los estados de ánimo, caracterizados por la impermanencia, los ámbitos cósmicos tampoco gozan de una estabilidad definitiva, aunque abarquen periodos de tiempo incomparablemente más extensos. El espacio cósmico o mundo receptáculo (bhājanaloka) experimenta ciclos recurrentes de repliegue o contracción desencadenados por tres de los elementos: fuego, agua y aire (Walshe, 1995, p. 345-350), ${ }^{34}$ que reducen los mundos de abajo a arriba, es decir, partiendo de los ámbitos de existencia más elementales y procediendo sucesivamente hacia los más evolucionados. El fuego destruye en primer lugar el ámbito de kāmadhātu, alcanzando el primero de los ámbitos de la materia sutil, correspondiente al primer dhyāna, donde se detiene. Los ámbitos superiores, correspondientes al segundo dhyāna, serán destruidos por el agua, mientras que los asociados al tercer dhyāna son destruidos por el viento. Únicamente los ámbitos correspondientes al cuarto dhyāna (siete pertenecientes al mundo de la materia sutil y cuatro al mundo inmaterial) no se ven afectados por este repliegue cósmico. Tanto el Visuddhimagga como el Abhidharmakośabhāsya describen detalladamente la secuencia y frecuencia de dicho repliegue cósmico. ${ }^{35}$ Tras siete ciclos de destrucción mediante el fuego sucede uno mediante el agua, lo que sugiere que la contracción cósmica no siempre tiene el mismo alcance.

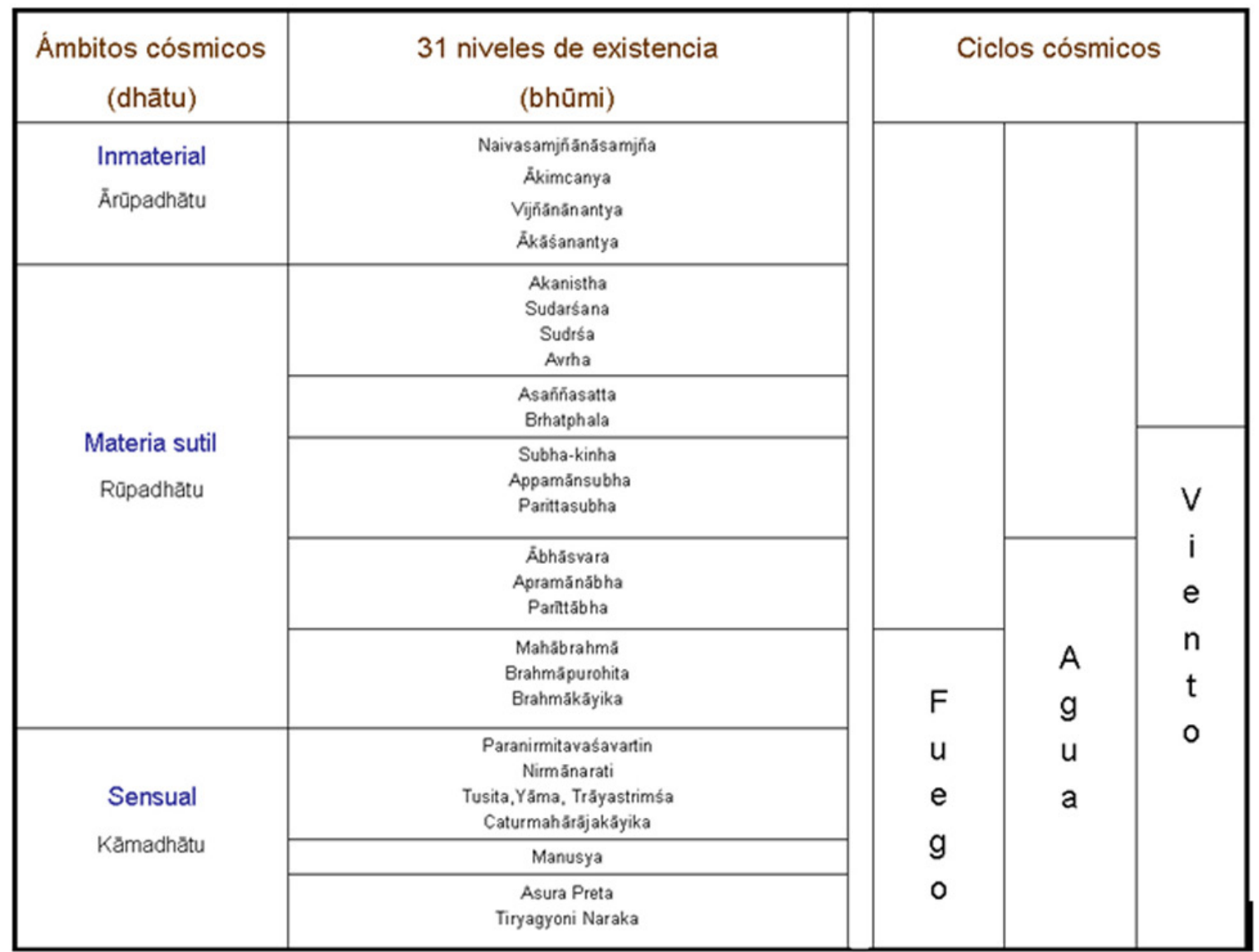


¿Qué ocurre con los seres de los ámbitos que son objeto del repliegue cósmico? Hay acuerdo en que dichos seres no pueden desvanecerse simplemente de samsāra, pero la escolástica del norte y la del sur no se ponen de acuerdo en cuál es su destino. Para Vasubandhu dichos seres renacen en ámbitos equivalentes de otros universos que no se encuentran en proceso de contracción, lo que preservaría la ley del karma. ${ }^{36}$ Sin embargo, para Buddhaghosa estos seres renacen en el mundo de la materia sutil, concretamente en el ámbito de ābhassara, en virtud de un karma positivo remanente. Yaśomitra, en su comentario al libro octavo de Kośa, sugiere que cuando se inicia el repliegue del universo, los seres de los ámbitos de existencia inferiores entran en el segundo dhyāna, gracias a una vāsanā o traza mental latente. Se subraya así la idea de que todos los seres en algún momento de su trayectoria han pasado por el ámbito de la materia sutil y que la contracción cósmica se encarga de actualizar. ${ }^{37}$

En la literatura antigua el término sánscrito kalpa hace referencia a periodos cósmicos de larga duración, asociados a los diversos ciclos de expansión y contracción del universo. El Abhidharmakośa distingue cuatro tipos de eras cósmicas o eones..$^{38}$ Una era de repliegue o contracción, llamada samvarta-kalpa, y una era de despliegue o expansión, llamada vivartakalpa. En función de su duración, se habla también de eras menores (antarakalpa) y mayores (mahākalpa). El mundo tiene una duración de veinte antarakalpa, una vez se ha consumido este tiempo, empieza la fase de repliegue (vivartakalpa), que se inicia, desde abajo, con la desaparición sucesiva de los ámbitos de existencia (gatisamvartanī) y sus receptáculos (bhājanasamvartanī). ${ }^{39}$

Ya hemos visto que mediante el agua se repliegan los ámbitos correspondientes al segundo dhyāna. En orden sucesivo: parittābha, appamānābha y ābhassara, todos ellos pertenecientes al mundo de la materia sutil. Mediante el viento se repliegan los ámbitos correspondientes al tercer dhyāna: el ámbito de parittasubha, el de appamānasubha y el de subhakinha. Los ámbitos superiores a este, asociados con el cuatro dhyāna, las llamadas moradas puras (suddhāvāsa) y los cuatro estados inmateriales (arūpya); no se repliegan y constituyen el universo latente cuando todo ha desaparecido. Podemos inferir que es en estos estados donde se custodian las diversas potencialidades de los seres, cuyos itinerarios kármicos sobreviven a las transformaciones cíclicas del cosmos. Dicha identidad kármica se hará efectiva en la era de despliegue o expansión (vivartakalpa), tras haber quedado en suspenso durante el periodo de transición entre el repliegue y el subsiguiente despliegue del cosmos. El funcionamiento descrito aquí para nuestro universo es extensible a otros universos, cuyo número es inimaginable.
El comentario explica el motivo por el cual los diversos elementos destruyen gradualmente los distintos ámbitos. El fuego destruye el primer dhyāna porque precisamente las imperfecciones y vicios de dicho estado mental son vitarka-vicāra, que hacen arder la mente del mismo modo que el fuego hace arder el mundo. El primer término, vitarka, hace referencia a la elucubración de lo discursivo, a suposiciones, conjeturas y dudas. El segundo, vicāra, al discernimiento y la reflexión. Ambos inquietan todavía al primer dhyāna. Los ámbitos asociados al segundo dhyāna, los esplendorosos (ābhāsvara), sucumben al agua, dado que su vicio es la dicha. Y los sūtra afirman que la sensación de sufrimiento se destruye mediante la supresión de la solidez del cuerpo, siendo los seres dichosos blandos y maleables como el agua. Los ámbitos asociados al tercer dhyāna los destruye el viento, pues tienen como vicio la respiración.

De modo que cada uno de los vicios asociados a los diferentes estados mentales causa la destrucción de los ámbitos de existencia. De nuevo lo externo y lo interno, lo físico y lo mental, se consideran dos aspectos de una misma realidad. ¿Por qué no se opera una destrucción mediante el elemento tierra?, se pregunta el autor: porque lo que llamamos mundo físico es precisamente el elemento tierra. Por lo tanto ese mundo a ese mundo físico puede oponerse el fuego, el agua o el viento, pero no la misma tierra.

Los ámbitos asociados al cuarto dhyāna no son objeto de destrucción precisamente porque su estado mental asociado se encuentra libre de la agitación de la especulación, la dicha y la respiración. ¿ Respeta esta concepción de un ámbito indestructible el principio de impermanencia que gobierna la especulación budista en torno al cosmos y la vida? Vasubandhu no elude la cuestión. El mundo receptáculo del cuarto dhyāna no es eterno, de hecho no constituye ni siquiera un ámbito que exista por sí mismo, sino que se divide en diferentes mansiones o residencias que surgen y perecen a medida que los seres las pueblan o abandonan. Aquí la distinción entre sattvaloka y bhājanaloka ha desaparecido. Dichos ámbitos existen siempre porque siempre se encuentran habitados, aunque ningún ser more eternamente en ellos, de hecho, no son ni siquiera espaciales. De modo que el cuarto dhyāna es y no es un sustrato permanente del universo. Lo es en el sentido de que funciona como su fuente de alimentación, como el resto donde se almacena la potencialidad kármica de los seres una vez el ciclo de repliegue ha concluido y el cosmos, suspendido, aguarda su despliegue postrero. ${ }^{40} \mathrm{Y}$ no lo es en el sentido de que las "identidades" que lo constituyen son fluctuantes e inestables. Dado que el ámbito de inmenso fruto (brhatphala) no constituye tanto un ámbito como un modo de ser. Decir que el mundo se contrae periódicamente hasta dichos modos de ser 
es una manera de decir, como apunta Gethin, que los seres retornan a lo que podría considerarse un modo primordial del ser y en este sentido dichos ámbitos pueden entenderse como un "origen" para el resto del cosmos (Gethin, 1997, p 204).

\section{EL DESPLIEGUE DEL COSMOS}

La era cósmica de despliegue o expansión, denominada vivarta-kalpa, se inicia con la aparición de viento primordial (prāgvāyu) y concluye cuando los seres comienzan a renacer en los ámbitos inferiores de kāmadhātu. ${ }^{41} \mathrm{El}$ mundo ha quedado en suspenso por largo tiempo, en concreto durante veinte kalpa menores (antarakalpa), habiendo sólo espacio donde antes se encontraban los seres. Por la fuerza remanente de su acción colectiva, aparecen los primeros signos de lo que será el mundo material. Una ligera brisa surge en el espacio vacío. Y se inicia la era de despliegue, que durará tanto como la de suspensión, veinte antarakal$p a$. La brisa primordial aumenta gradualmente de intensidad hasta formar un círculo de viento. De esta manera se forman todos los "receptáculos" que albergarán los ámbitos de existencia (bhūmi). Los primeros en poblarse son los ámbitos de rūpadhātu y en orden descendente se van poblado los diversos bhūmi hasta llegar al más bajo de todos ellos. Cuando esto ocurre, la era de despliegue ha finalizado. En la primera se crea el mundo físico, en las otras diecinueve, hasta que los seres renacen en los abismos, la vida humana goza de una incalculable longevidad, que va disminuyendo conforme avanza la evolución del cosmos. ${ }^{42}$

\section{CONCLUSIONES}

De lo expuesto hasta aquí hay varias ideas que pueden resultar atractivas para la sensibilidad moderna. Por un lado, según el entendimiento budista del cosmos, el tiempo se concibe como la distancia entre la mente y el universo. Por el otro se subraya la idea de que el universo es un mapa de la mente: el complejo itinerario de los estados de ánimo. La cosmología budista, a diferencia de la actual, no se organiza exclusivamente en torno a lo sensible (la bóveda estrellada, digamos), sino en torno a los diversos estados de la experiencia mental. "El ojo sólo percibe lo visible, el oído lo audible, y nada sabe uno del otro, mientras que la mente (manas) aprende tanto su objeto, la conciencia, como los objetos de los cinco sentidos y los sentidos mismos" (Bodhi, 2002, p. 325). ${ }^{43}$ Desde el punto de vista budista, es lógico que la cosmología moderna se organice en torno a lo sensible pues es un conocimiento desarrollado en el mundo sensual de kāmadhātu.
En la cosmología budista el espacio no se considera como una categoría plausible al margen de una percepción cognitiva que de alguna manera lo impregna y configura. De modo que considerar un espacio vacío de percepción supondría una contradicción en los términos. Curiosamente, la tradición que hizo del vacío una categoría filosófica, descartó o pasó por alto la posibilidad de que el espacio pudiera existir al margen de la percepción, es decir, al margen de los seres que lo habitan, ya sean corrientes mentales toscas y sensibles (kāmadhātu), sutiles (rūpadhātu) o inmateriales (ārūpadhātu). Al ser función de los estados de la mente, el espacio pierde su indiferencia y se atempera, se convierte en una actitud y un modo de ser, se dota de inclinaciones. Y la curvatura del espacio, su gravedad, se torna función de las miradas que lo crean. Así, la tradición hablará con frecuencia de espacios inquietos y espacios serenos, espacios oscuros y luminosos, ámbitos de dicha y ámbitos de sufrimiento.

El universo no tiene origen, pero se repliega y despliega cíclicamente. Cada ciclo se compone de cuatro fases. En la fase de expansión se produce una diversificación unida a una sucesiva degradación en la experiencia de los seres. En la fase de contracción el universo comienza a recogerse desde abajo, desde los niveles más bajos de conciencia hasta los más elevados, que son los últimos en reabsorberse. Desde esta perspectiva, si consideramos lo que ocurre en un único ciclo de despliegue-repliegue, puede decirse que son los estados de conciencia que han logrado liberarse de la atadura de la materia (el mundo de ārèpyadhātu), los que permanecen como universo embrionario cuando éste se encuentra en su ciclo de "suspensión", y es a partir de ellos, cuando se inicia de nuevo el despliegue cósmico, donde se forma primero el mundo de la materia sutil (o de refinada sensibilidad) y, posteriormente, el mundo de sensibilidad tosca en el que habitamos. Según esta narrativa, la conciencia, o mejor, los estados de conciencia, crean el receptáculo para lo sensible. La mente se crea un cuerpo.

Pero dicha narrativa puede invertirse si nos situamos en el inicio del proceso de repliegue, y lo que era entonces efecto se convierte ahora en causa. Según esta otra perspectiva, el repliegue de lo sensible y de lo material da paso a un mundo imperceptible que carece de forma pero no de entendimiento, donde los estados de conciencia subsisten al margen de lo material. 


\section{NOTAS}

1 Brahmajalasutta (Dīghanikāya: 1). Véase Tola y Dragonetti (2010) y Walshe (1995)

2 Otros diálogos de los nikāya y āgama confirman esta actitud. La especulación en torno a la naturaleza del cosmos, su origen y evolución, se consideraba perniciosa y desorientadora. La extensión espacial y temporal del universo se plantea también en el Diálogo breve con Mālunkya, donde el eremita Mālunkya, impaciente por que el Buda le aclare esta y otras cuestiones, va a interrogarlo a los jardines de Anāthapindika, en los alrededores de Śrāvasti. El Buda elude la cuestión por no ser provechosa al propósito de la enseñanza: la erradicación del sufrimiento, y por no conducir a la serenidad, el desapego y la ecuanimidad. La naturaleza del espacio y del tiempo no son cuestiones prioritarias, y se ilustra mediante la alegoría del hombre que, herido por una flecha envenenada, se resiste a dejarse curar hasta conocer la identidad del arquero. Vano es preguntarse por la finitud o infinitud del universo, por los límites del espacio o del tiempo: dichas especulaciones alejan al monje del verdadero propósito de la doctrina. Cūlamālunkyasutta (Majjhimanikāya 63), (Ñanamoli y Bodhi, 1995).

3 Kośa IV-1a

4 Desoyendo las recomendaciones del Maestro, un monje de nombre Kevata, se recreaba en inquisiciones sobre el origen del mundo. Con el objeto de saber dónde se disuelven los cuatro elementos (donde termina el espacio o, en términos budistas, donde acaba el mundo condicionado), Kevata asciende mediante la meditación a los diversos ámbitos de existencia para interrogar a sus moradores. A todos ellos pregunta y ninguno de los interrogados sabe responderle. Finalmente Kevata llega ante Brahmā y formula impaciente su pregunta. El dios, ceremonioso y autocomplaciente, elude reconocer su ignorancia, aunque finalmente le aconseja que regrese junto al Buda y le pregunte a él. La respuesta que obtiene del Buda confirma la correspondencia entre espacio físico y estado mental. Los cuatro elementos no cesan "ahí fuera", el algún remoto lugar del cosmos, sino en la propia conciencia. El relato es un buen ejemplo de las concepciones mentales del espacio físico, donde diferentes estados de la mente cultivada sirven de acceso a diversos ámbitos cósmicos. Según esta lectura, el mapa del cosmos constituye un detallado informe de todas las experiencias posibles por las que transita la vida consciente (Dragonetti, 1984, pp. 101-114).

5 Ko§a III 1a-c, p. 365

6 Ko§a III 1c-d, p. 365.

7 Anguttaranikāya: 3, 411. Ref, (Vallée-Poussin, 1908, p.130).

8 Koßa III 3c-d, p.367.

9 En las teorías budistas sobre la identidad personal, el primero de los skandha es nama-rūpa. En este contexto rūpa hace referencia al cuerpo material del individuo, mientras que nāma se refiere al complejo psíquico constituido por la percepción (samjñā), la sensación (vedanā), las inclinaciones (samskāra) y la apercepción (vijñāna).

10 Koßa III 2 a-d.

11 El esquema de los dhyāna fue desarrollándose gradualmente. A veces se consideran ocho, siendo el cuarto subdividido en otros cuatro.
12 La experiencia de los dhyāna no se considera un fin en sí mismo sino un medio para la obtención del nirvana. Los dhyāna son estados momentáneos y condicionados, por tanto insatisfactorios. Una vez se sale de ellos la mente recobra sus carencias.

13 Kośa III 2. Aunque la paramnesia aquí aludida no implica un desorden de la memoria, como supone en la medicina moderna. El primer dhyāna se asocia con el ámbito Brahmakāyika, Brahmapurohita y Mahābrahma. El segundo con Parīttābha, Apramānābha y Abhāsrava. El tercero con Parīttaśubham Apramānaśubha y êubhakrtsna. El cuarto con Anabhraka, Punyaprasava, Brhatphala y los cinco Śuddhāvāsika: Avrha, Atapas, Sudrśa, Sudarśana y ākanistha. Estos diecisiete ámbitos (bhūmi) constituyen el mundo de rūpadhātu, aunque otras escuelas como la de Cachemira listan sólo dieciséis.

14 Ko§a III 3a, p. 365.

15 ākāśānantyāyatana, vijñānantyāyatana, ākimcanyātana y naivasamjñāyatana.

16 Kośa III 3c-d. La Vallée Poussin (1988, p. 367) comenta: La mente y los estados mentales de los primeros dos dhātu son āßrita (algo soportado) y tienen como soporte un cuerpo dotado de órganos. La mente "cae" o muere, cuando se destruyen dichos órganos.

17 Ko§a II 41a.

18 Kośa VIII 3, p. 1220

19 Kośa VIII 19 c-d.

20 Saxyutta nikāya: 34. 7, en (Bodhi, 2002).

21 Kośa VIII 1 a-d.

22 Kośa VIII 2 a-b, p. 1218

23 Kośa VIII 2 c-d, p. 1219-20.

24 Kośa I. 38 c-d, p. 106

25 Kośa VIII, 3c, p. 1225

26 Kośa VIII, 3d, p. 1225-26.

27 Kośa, p. 1287 nota 41

28 Majjhima nikāya: 121, en Ñanamoli y Bodhi (1995).

29 Visuddhimagga XIII, en Ñanamoli (1999).

30 Visuddhimagga XIII 40, p. 412

31 Visuddhimagga XIII 28, p. 410

32 Visuddhimagga XIII 29, p. 410

33 Visuddhimagga XIII 30, p.410.

34 Aggaññasutta, Dīghanikāya: 27. 
35 Visuddhimagga XIII 55-62, 65, pp. 414-417, Kośa: III 102, p.495.

36 Kośa III 90 a-b, p.475-477.

37 Kośa VIII 38 c-d, p.1280.

38 Kośa III 89 d, p. 475.

39 Kośa III 90 a-b, pp.475-477. Otras fuentes budistas barajarán la idea de un "Eón inconmensurable" (asamkhyeya-kalpa), cifrado, según las diversas fuentes, en $10^{51}, 10^{59}, 10^{63}$ años, añadien-

\section{BIBLIOGRAFÍA}

Bodhi, Bhikkhu (trad.) (2002), The Connected Discourses of the Buddha: A Translation of the Samyutta Nikaya, Boston, Wisdom Publications.

Dragonetti, Carmen (trad.) (1984), Siete sètras del Dïgha Nikāya, diálogos mayores del Buddha, México D. F., El Colegio de México.

Gethin, Rupert (1997), "Cosmology and Meditation. From the Aggañña-Sutta to the Mahāyāna", History of Religions, (36) 3, pp. 185-204.

Ñanamoli, Bhikkhu (1999), The Path of Purification by Buddhagho$s a$, Seattle (WA), BPS Pariyatti Editions.

Ñanamoli, Bhikkhu y Bodhi, Bhikkhu (1995), The Middle Length Discourses of the Buddha: A Translation of the Majjhima Nikaya, Boston, Wisdom Publications. do que un bodhisattva se convierte en Buda tras tres de estas inconmensurables eras (Kośa III 93d, 94a, pp.479-480).

40 Siempre que no se descarte la transferencia a otros universos para preservar la conservación del karma.

41 Kośa 3.90 c-d, p. 477.

42 Kośa iii 91 a-b, p. 478.

43 Samyuttanikāya V.

Tola, Fernando y Dragonetti, Carmen (2010), Diálogos mayores de Buda, Madrid, Trotta.

Vallée-Poussin, Louis (1908), "Cosmogony and Cosmology (Buddhist)". En Hastings (ed.), Enciclopaedia of Religions and Ethics, IV, p. 130.

Vallée-Poussin, Louis (ed./trad.) (1988), Abhidharmakośabhāsyam, Berkeley (CA), Asian Humanities Press.

Walshe, Maurice (1995), The Long Discourses of the Buddha. A Translation of the Dïgha Nikāya, Boston, Wisdom Publications. 